\title{
Sensor Data Fusion Using Mutual Information Algorithm
}

\author{
Mahdieh Shabanian ${ }^{1 *}$, Seyed Hadi Hosseini ${ }^{1}$ \\ ${ }^{1}$ Department of Electrical Engineering, East Tehran Branch, Islamic Azad University, \\ Tehran, Iran
}

\begin{abstract}
Traffic flow prediction is one of the congestion avoidance methods in highways. According to previous studies, no comprehensive model has been proposed for traffic flow prediction which can prevent congestion in many different traffic conditions. Using data fusion to reduce prediction error is an interesting idea to solve this problem. In this paper, a new hybrid algorithm based on mutual information for traffic flow prediction will be proposed and compared with various types of previous hybrid algorithms and predictors. The Mutual Information (MI) algorithm is used to calculate the interdependency of data, so we expect this new hybrid algorithm to have high precision in comparison with others. Simulations will be implemented based on real data in MATLAB environment as a performance demonstration of new hybrid algorithm. Due to variety of traffic flow, performance investigations of our new hybrid algorithm will be done in presence of polluted traffic data in different climatic conditions such as rain/snow fall or other traffic conditions like congestions and accidents on the road, indicating robustness of this algorithm to different types of noisy data.
\end{abstract}

Keywords: Fusion Algorithm, Hybrid Models, Traffic Flow Prediction, Mutual Information Algorithm. 


\section{Introduction}

\subsection{Statement of the problem}

$\mathrm{I}$ ncreasing of social activities causes the problems in transportation, especially in the road and big cities highways. The transportation systems need an intelligent management system using advanced technologies to improve their efficiency in control and manage of complicated problems. Accordingly, the Intelligent Transportation Systems (ITS) has been developed in recent years. The ITS using the information theory, telecommunications and control technologies and system engineering, could solved very traffic problems. Reducing fuel consumption, air pollution, traffic congestion, travel time and enhancing effectiveness of social and economic activities are some of effective results of ITS exploitation in road transportation system. Traffic flow prediction is one of the solutions that proposed by ITS for congestion prevention in highways.

\subsection{Literature Review of Traffic Flow Prediction}

Traffic flow is completely nonlinear, stochastic process and considered one of the macroscopic features of traffic. Nowadays, according to previous researches in traffic flow prediction, most of traffic flow prediction methods are empirical and data-based, so they can be categorized into three main groups: parametric, nonparametric and hybrid methods.

\subsubsection{Parametric Prediction Methods}

In most parametric prediction methods using regression models (Sun, 2003), maximum likelihood model (Ramezani, 2012) and time series models such as ARIMA (Tan, 2009), ARIMAX (Williams, 2001) and SARIMA (Williams, 2003), all previous data are modeled and the traffic flow of forward steps can be predicted. Simplicity of prediction model is an advantage of these methods and dependency to a lot of previous data and also poor performance in presence of noise, disturbance and sudden intense variations are major disadvantages of them.

\subsubsection{Nonparametric Prediction Methods}

Certain traffic phenomena such as congestion or accident on the road are predictable more precisely in nonparametric prediction methods. Likewise, model complexity and high dependency to a lot of data are the most significant disadvantages of them. Neural networks such as MLP, RBF and TDNN (Bin, 2006, Nagare, 2012), K Nearest Neighborhood (Chang, 2012) and SVM models (Castro-Neto, 2009) are among the most common nonparametric prediction methods that have been used more often for traffic flow prediction.

\subsubsection{Hybrid Prediction Methods}

Proper solutions are just given in certain conditions by each of pre-mentioned methods and in the case of changing traffic conditions, they will not robust and maintain their high efficiency in traffic flow prediction. Researchers have utilized linear or nonlinear combination of parametric and nonparametric prediction methods in recent years and developed hybrid prediction methods whose accuracy depends on combination process type associated with parametric and nonparametric methods used. Of course these methods are more complex than other two and their implementation are costly (Hosseini, 2014). A hybrid of neural network with other prediction models like fuzzy model (Stathopoulos, 2008), wavelet model (Jiang, 2005) and ARIMA model (Zeng, 2008) are one of these methods. Actually no comprehensive method has been proposed yet for traffic flow prediction which could lead to very accurate solutions in all traffic conditions (Stathopoulos, 2008).

\subsection{The combination of models}

One prediction model itself can have high prediction quality in certain conditions but probably not in other traffic conditions; so 
combination of multiple different prediction models each suitable for various traffic conditions can solve this issue (Hosseini, 2012). The hybrid prediction models typically have high accuracy in most different traffic conditions and with any kind of data; structure flexibility is their other characteristic (Zhang, 2012). Different types of simple parametric and nonparametric prediction models can be combined using various hybrid algorithms to develop a new hybrid model.

First hybrid algorithms of multiple models which combined few models in form of linear combination return to late 1970s, worked separately by Chong and Spyer on optimal expanded kalman filters (Chong, Spyer, 1979) and later changed to a unique model (Li, 2003). Then in late 1980s, hybrid algorithms expanded upon nonlinear systems, too $(\mathrm{Xu}, 2003$, Torra, 2007). Arithmetic Averaging (AA), Weighted Arithmetic Averaging (WA), Ordered Weighted Averaging (OWA) and Error-based Weighted Arithmetic Averaging (EWA) algorithms are very popular examples of hybrid algorithms $(\mathrm{Xu}$, 2003, Torra, 2007). In this paper, the AA hybrid algorithm that only applying arithmetic averaging on results of prediction models and also the EWA hybrid algorithm that utilizing prediction error rate of prediction models to determine their weighting factors in hybrid models, are used as comparative models. According to disadvantages of both algorithms and to solve them, new hybrid algorithm that determining weighting factors of prediction models using Mutual Information (MI) algorithm will be proposed and analyzed. Using MI algorithm in every combination and in accordance to type of input prediction models will enhance prediction quality. In the following we will predict traffic flow with developed hybrid algorithm using data related to certain traffic conditions such as traffic congestion, accident on the road and awful climatic conditions like heavy rain or snow, and then analyze outcomes.

The remainder of the paper is organized as follows: in section 2 mutual information algorithm is explained. Section 3 explains hybrid algorithms of pre-mentioned prediction models and subsequently introduces a new hybrid algorithm based on mutual information algorithm. Case study on traffic data and traffic flow prediction along with simulations are included in section 4 and finally section 5 concludes this paper.

\section{Mutual Information}

In control systems, the amount of MI is used to measure the nonlinear interdependence of two random variables. In other words, the amount of MI determines the amount of information on random variable $X$ obtained from the random variable $\mathrm{Y}$. that is denoted as $\mathrm{I}(\mathrm{X} ; \mathrm{Y})$ and calculation of MI helps to reduce uncertainty of $X$ while $Y$ occurs (Williams, 2009). The following equations and the Venn diagram in Figure 1 illustrate the MI amount between two random variables $X$ and $Y$ and their entropy. $H(Y)$ represents the entropy of $Y$ and $H(Y \mid X)$ shows the conditional entropy level.

$\mathrm{I}(\mathrm{X} ; \mathrm{Y})=\mathrm{H}(\mathrm{Y})-\mathrm{H}(\mathrm{Y} \mid \mathrm{X})$

$H(X)=-E\{\log P(X)\}=-\int P(X) \log P(X) d x$

$\mathrm{H}(\mathrm{X} \mid \mathrm{Y})=-\mathrm{E}\{\log \mathrm{P}(\mathrm{X} \mid \mathrm{Y})\}=$

$\mathrm{I}(\mathrm{X} ; \mathrm{Y})=\mathrm{I}(\mathrm{Y} ; \mathrm{X})$

$$
-\iint \mathrm{P}(\mathrm{X} \mid \mathrm{Y}) \log \mathrm{P}(\mathrm{X} \mid \mathrm{Y}) \mathrm{dx} d \mathrm{~d}
$$

$\mathrm{I}(X ; X)=\mathrm{H}(X)$

$\mathrm{H}(\mathrm{X}) \geq 0$

Where; $P(X)$ noted the probability density function of $X, P(X \mid Y)$ the probability density function of $X$ given $Y$, and $E$ means expected value operator. By substituting Equation (2) and Equation (3) in Equation (1), MI will be defined in Equations (7) and (8) in continuous or discrete forms (Hosseini, 2014).

$\mathrm{I}(\mathrm{X} ; \mathrm{Y})=\iint \mathrm{P}(\mathrm{x}, \mathrm{y}) \log \{\mathrm{P}(\mathrm{x}, \mathrm{y}) /(\mathrm{P}(\mathrm{x}) \mathrm{P}(\mathrm{y}))\} \mathrm{dxdy}$

$\mathrm{I}(\mathrm{X} ; \mathrm{Y})=\sum \sum \mathrm{P}(\mathrm{x}, \mathrm{y}) \log \{\mathrm{P}(\mathrm{x}, \mathrm{y}) /(\mathrm{P}(\mathrm{x}) \mathrm{P}(\mathrm{y}))\}$

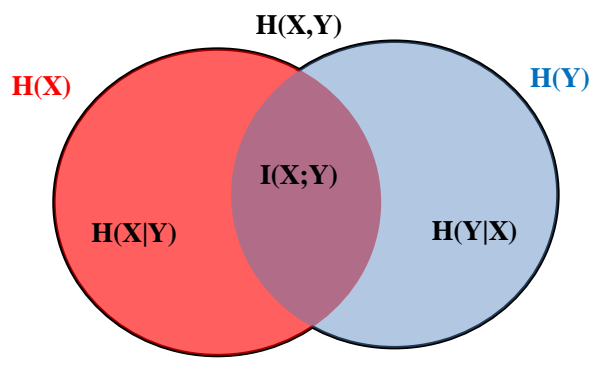

Figure 1: The representation of mutual information and different entropies between $X$ and $Y$ 
Here, $P(x, y)$ represents the probability density function for $X$ and $Y$ random variables. Thus it is sufficient to know $\mathrm{P}(\mathrm{x}, \mathrm{y})$ to obtain $\mathrm{I}(\mathrm{X} ; \mathrm{Y})$. This function is, however, unknown and therefore needs to be estimated (Hosseini, 2014). According our previous works and based on the definitions and equations are denoted in (Hosseini, 2014), the MI estimation is:

$$
\begin{aligned}
\hat{\mathrm{I}}(\mathrm{X} ; \mathrm{Y})= & \Psi(\mathrm{k})-\left(\frac{1}{\mathrm{k}}\right)- \\
& \left(\frac{1}{\mathrm{n}}\right) \sum_{\mathrm{i}=1}^{\mathrm{n}}\left(\Psi\left(\mathrm{n}_{\mathrm{i}}^{\mathrm{x}}\right)+\Psi\left(\mathrm{n}_{\mathrm{i}}^{\mathrm{y}}\right)\right)+\Psi(\mathrm{n})
\end{aligned}
$$

where, the function $\Psi$, represent the Digamma function respectively (Hosseini, 2014).

\subsection{MIFS Algorithm}

In real systems, a significant degree of uncertainty occurs in the identification system output due either to inadequacy of initial data or suboptimal condition of the system. Since the inadequacy of initial data in traffic systems is ruled out, the main reason for the existing uncertainty can be attributed either the sensing system or the noisy pollution of the data. Selecting the appropriate and adequate data from among a great pool of data can be a working solution for this problem. In this paper, an algorithm to select the best input data has been used to identify an optimal subset of initial candidate input data. Various algorithms exist for the selection of efficient inputs, notably Battiti's Mutual Information Feature Selection (MIFS) algorithm. In MIFS algorithm, the aim is to obtain a relationship between the inputs and the output in order to reduce the existing redundancy in the input data and at the same time select the data with highest relationship with the output (Battiti, 1994).

\subsubsection{The steps of MIFS algorithm}

At first, the output set of $\mathrm{T}$ and $\mathrm{m}$-member set of $\mathrm{L}$ are supposed that it includes li's. In addition, we assume an empty set of $S$ with no data. Then should obtained the mutual information for each input li member of $\mathrm{L}$ and compute the I(li;T). We select the lj input which maximizes the information with output $\mathrm{I}(\mathrm{lj} ; \mathrm{T})$ and then separate it from the set L and add it into set $\mathrm{S}(\mathrm{s} 1=\mathrm{lj})$. We repeat these steps until all inputs of $\mathrm{L}$ are selected. Then as Equation (10), we have two stages. At first for all couples of variables (li, s) with li member of $L(i \neq j)$ and $s m$ member of $S$, the I(li ;sm) is computed. Then we select the li input which maximizes the following term and separate it from the set L and add it into set $S$.

$\operatorname{Max}_{i}\left\{\mathrm{I}(\mathrm{li} ; \mathrm{T})-\beta \sum \mathrm{m} \mathrm{I}(\mathrm{li} ; \mathrm{Sm})\right\}$

The important parameter to consider is $\beta$, which shows the amount of augmentation between the inputs. In the present paper, empirically $\beta$ is chosen to be 0.6 .

\section{Data Fusion Systems}

\subsection{Data Fusion Architectures}

As the previous research we can divide the structure of data fusion systems in to three: centralized, distributed and fusion architecture (Durrant-Whyte, 2001).

\subsubsection{Centralized Fusion Architecture}

In this architecture, the main fusion center is located in the central process unit and all decisions were taken in there. This architecture is shown in Figure 2.

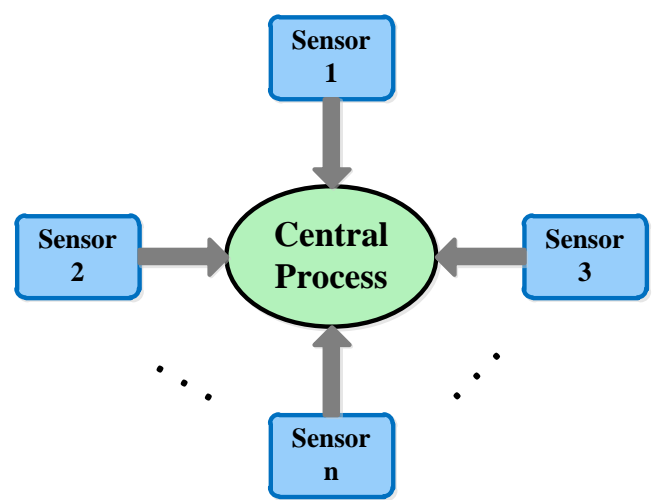

Figure 2: The structure of centralized fusion architecture

\subsubsection{Distributed Fusion Architecture}

In this architecture, the measured values of each sensor individually processed and refined and eventually all the useful information is sent to a central processor. Then the central processor 
fused the information transmitted from distributed processor with the other sensors data. This architecture can be considered as a developed form of central fusion architecture. Its structure is shown in Figure 3.

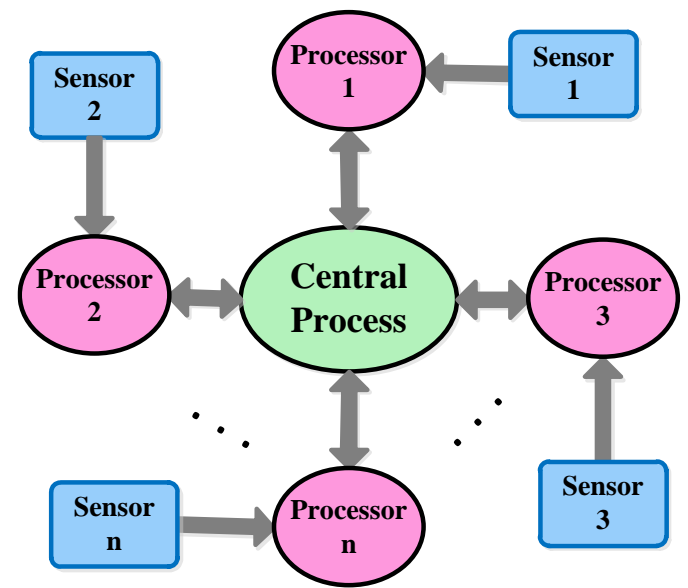

Figure 3: The structure of distributed fusion architecture

\subsubsection{Hybrid Fusion Architecture}

By combining two previous architectures a new architecture is achieved that known as hybrid fusion architecture. Generally, the hybrid architecture has high accuracy in the different conditions with any type of data. This structure is really flexible and using different algorithms the variety of centralized and distributed architectures are fused and the new hybrid architectures invented. In Figure 4, the structure of one hybrid architecture is shown.

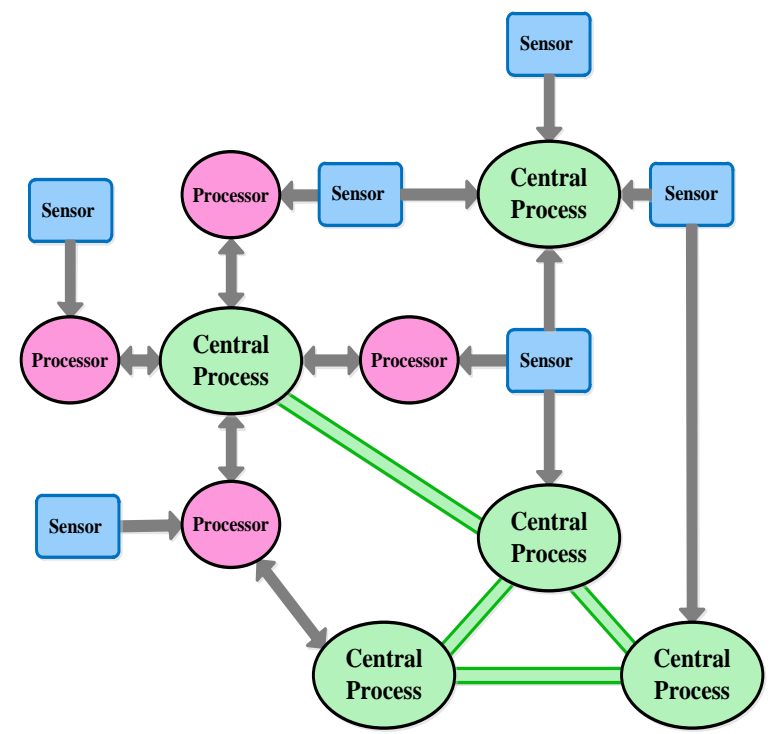

Figure 4: The structure of hybrid fusion architecture
In this paper, for all discussed fusion models the centralized architecture will be used.

\subsection{Data Fusion Algorithms}

Traffic data used in literature are related to traffic flow with 15-minute time intervals between data. Considering data discontinuity, $\left\{X_{t}\right\}$ time series can be supposed as traffic flow data (sensor observations) and one step ahead prediction problem as Equation (11).

$X_{\hat{t}}=f\left(X_{(t-1)}, X_{(t-2)}, X_{(t-3)}, \ldots, X_{(t-m)}\right)$

which; $\mathbf{X} \mathfrak{t}$ indicates predicted traffic flow at time t.

In the following, firstly we explain AA and EWA fusion algorithms and then will propose and evaluate new MI fusion algorithm in order to alleviate their disadvantages.

\subsubsection{AA Data Fusion Algorithm}

The AA algorithm is the simplest fusion algorithm that used as a benchmark in the recent researches and references (Alexandre, 2001). As a result of Equation (12), all prediction models have same value in this algorithm and final result is arithmetic average of them. In case of poor performance for one set of data, the overall performance of the algorithm will be low. So the most significant disadvantage of AA algorithm is inability to accurately recognize the data and assign same value to all of them in combination process.

$\widehat{\mathbf{X}}_{\mathrm{t}}=\frac{1}{\mathrm{~m}} \sum_{\mathrm{i}=1}^{\mathrm{m}} \mathrm{X}_{\mathrm{t}-\mathrm{i}}$

\subsubsection{EWA Data Fusion Algorithm}

Unlike AA algorithm in EWA algorithm the value of every data set is different and their error rate determines their weighted factors in combination process. Equation (13) represents weighted factor ai for each of data set; their corresponding values will not be the same. Calculation procedure of weighted factors for EWA algorithm is included in Equation (14). Hence, a set of data with more prediction error variance has negligible cooperation in combination process and vice versa. 


$$
\begin{gathered}
\widehat{\mathbf{X}}_{\mathrm{t}}=\frac{\sum_{\mathrm{i}=1}^{\mathrm{m}} \mathrm{a}_{\mathrm{i}} \mathrm{X}_{\mathrm{t}-\mathrm{i}}}{\sum_{\mathrm{i}=1}^{\mathrm{m}=1} \mathrm{a}_{\mathrm{i}}} \\
\mathrm{a}_{\mathrm{i}}=\left(\sigma_{\mathrm{i}}^{2}\right)^{-1}=\frac{1}{\sigma_{\mathrm{i}}^{2}}
\end{gathered}
$$

The term $\sigma_{i}{ }^{2}$ in Equation (14) indicates prediction error variance of $i^{\text {th }}$ set data at time $t$. In EWA, weighted factors of each data set depends only on prediction error variance of that model and is not much affected by other ones' results; this is the most important disadvantage of EWA and makes us propose new MI fusion algorithm.

\subsubsection{MIWA Data Fusion Algorithm}

In the previous section MI algorithm introduced and the process of its calculation explained. Now using this algorithm new data fusion algorithm introduced named MIWA algorithm. In this new algorithm using MI the weighting factor of any data set is calculated. In detect of the days that have little impact on the prediction result and delete them, the MIWA algorithm is able to reduce the volume of calculations and prediction error at the same time. The MIWA algorithm used the Equation (13) for combining data from the past days and to determine of their weighted factor we use MI algorithm. Unlike EWA method, in MIWA fusion algorithm only $\mathrm{k}$ appropriate data set from $\mathrm{m}$ data set prioritized and selected. The calculation procedure is expressed in Equation (15).

$$
\begin{aligned}
& \mathrm{I}\left(\mathrm{X}_{\mathrm{t}} ; \mathrm{X}_{\mathrm{t}-\mathrm{i}}\right)=? \quad ; \mathrm{i}=1,2, \ldots, \mathrm{m} \\
& \left\{\begin{array}{c}
\mathrm{L}-\left\{\mathrm{X}_{\mathrm{t}-\mathrm{i}}\right\} \rightarrow \mathrm{L} \\
\left\{\mathrm{X}_{\mathrm{t}-\mathrm{i}}\right\} \rightarrow \mathrm{S}
\end{array} ; \mathrm{i}=\arg \left\{\max _{\mathrm{i}}\left(\mathrm{I}\left(\mathrm{X}_{\mathrm{t}} ; \mathrm{X}_{\mathrm{t}-\mathrm{i}}\right)\right)\right\}\right. \\
& \text { for } \mathrm{d}=1,2, \ldots, \mathrm{k} \rightarrow\left\{\begin{array}{l}
\mathrm{i}=1,2, \ldots, \mathrm{m}-\mathrm{d} \\
\mathrm{j}=1,2, \ldots, \mathrm{d}
\end{array}\right. \\
& \max _{\mathrm{X}_{\mathrm{t}-\mathrm{i}}}\left\{\mathrm{I}\left(\mathrm{X}_{\mathrm{t}} ; \mathrm{X}_{\mathrm{t}-\mathrm{i}}\right)-\beta \sum_{\mathrm{j}} \mathrm{I}\left(\mathrm{X}_{\mathrm{t}-\mathrm{i}} ; \mathrm{s}_{\mathrm{j}}\right)\right\}=? \\
& \Rightarrow\left\{\mathrm{X}_{\mathrm{t}-\mathrm{i}}\right\} \rightarrow \mathrm{S} \\
& \mathrm{S}=\left\{\mathrm{s}_{1}, \mathrm{~s}_{2}, \ldots, \mathrm{s}_{\mathrm{k}}\right\} \subset \mathrm{L}
\end{aligned}
$$

According to the result of Equation (15) the selected s1 data set is the highest accuracy and sk data set is the least accuracy between $\mathrm{k}$ data set. Then $\mathrm{s}_{1}$ data set is first priority to participate in fuse operation and $\mathrm{sk}$ data set is $\mathrm{k}^{\text {th }}$ priority. According to the priority for $\mathrm{k}$ selected data sets, the MIWA algorithm determines their weighting factors with the use of numbers from 1 to k. For example the weighting factors of first priority selected data set is $\mathrm{k}(\mathrm{a} 1=\mathrm{k})$, the second priority selected data set is $\mathrm{k}-1\left(\mathrm{a}_{2}=\mathrm{k}-1\right)$ and least priority selected data set is $1 \quad\left(a_{k}=1\right)$. The calculation procedure of weighted factors for MIWA algorithm is stated in Equation (16).

$a_{i}=k+1-i \quad ; i=1,2, \ldots, k$

By placement the Equation (16) in Equation (13), the procedure of MIWA fusion algorithm is achieved that be stated in Equation (17).

$\widehat{\mathbf{X}}_{\mathrm{t}}=\frac{\sum_{\mathrm{i}=1}^{\mathrm{k}} \mathrm{a}_{\mathrm{i}} \mathrm{X}_{\mathrm{t}-\mathrm{i}}}{\sum_{\mathrm{i}=1}^{\mathrm{k}} \mathrm{a}_{\mathrm{i}}}=\frac{\mathrm{ks}_{1}+(\mathrm{k}-1) \mathrm{s}_{2}+\cdots+1 \times \mathrm{s}_{\mathrm{k}}}{1+2+\cdots+\mathrm{k}}$

\section{Experiment Setup and Case Study}

In this paper to verify new MIWA fusion algorithm, we perform two simulations in normal and abnormal traffic conditions and MATLAB environment besides showing dominance of new algorithm in recognizing these conditions. Abnormal traffic conditions mentioned here are related to heavy traffic as a consequence of accident. In order to compare algorithms, prediction error rate is calculated using three error criteria: mean absolute error (MAE), mean absolute percent error (MAPE) and variance absolute percent error (VAPE) as the following equations.

MAE: mean $\left(\sum \mid\right.$ Real Value - Prediction Value $\left.\mid\right)$

MAPE: mean $\left(\left(\sum \mid\right.\right.$ Real Value -Prediction Value $\left.\mid\right)$

$$
/(\text { Real Value })) \times 100 \%
$$

VAPE: variance $\left(\left(\sum \mid\right.\right.$ Real Value -

Prediction Value I) / (Real Value)) $\times 100 \%$ 


\subsection{Used data}

Data used for simulations are derived from mounted sensors on highway network of Metro in Minnesota, available online (http1). The paper uses information of three loop detectors called d783, d784 and d785 mounted on station No.286 (After ramp out) of EB highway, I-394 (west to east) as shown in Figure 5. The data refer to two months of 2012 (April and June) and the available real time traffic flow has been derived in a 15-minute time interval using special software (http2). Our predictions in this paper rely on data related to work days; we are to predict a-step-forward and traffic flow of 15 minutes later.

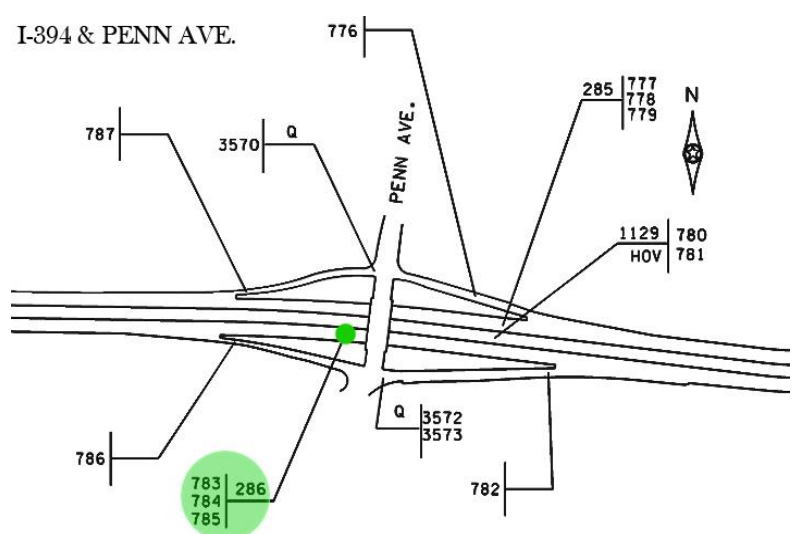

Figure 5: Map of the station No. 286 and detectors 783,784 and 785 are located in highway I-394 (http1)

\subsection{Simulation 1: Prediction using Normal Data}

In this simulation our goal is to predict traffic flow using some data related to normal days without any disturbance and also to evaluate performance of previous fusion algorithms. Data used refer to June 2012; traffic flow prediction of $29^{\text {th }}$ June (Friday) using data of past twenty work days in June is of interest. AA fusion algorithm calculates arithmetic average of previous twenty workday's data and finally predicts traffic flow for next step. EWA fusion algorithm can measure variance of prediction error of these data in every step, produces their corresponding weighted factors considering error variances and predicts traffic flow using weighted combination of them at last. MIWA fusion algorithm as a selective model considers output results of just previous six workday's data ( $\mathrm{k}$ is empirically select to be 6) at every moment, determines their suitable weighted factors and combines them which leads to a better predicted output. Results of this simulation are depicted in Figure 6 and Table 1.

Table 1: Traffic flow prediction error for different data fusion algorithms in simulation 1

\begin{tabular}{c|c|c|c}
\hline \multirow{2}{*}{ Simulation 1 } & \multicolumn{3}{|c}{ Data Fusion Algorithms } \\
\cline { 2 - 4 } & AA & EWA & MIWA \\
\hline MAE & 49.7156 & 40.0178 & 32.8705 \\
\hline MAPE (\%) & 9.5039 & 7.8832 & 6.0078 \\
\hline VAPE (\%) & 1.0127 & 0.8437 & 0.3971 \\
\hline
\end{tabular}

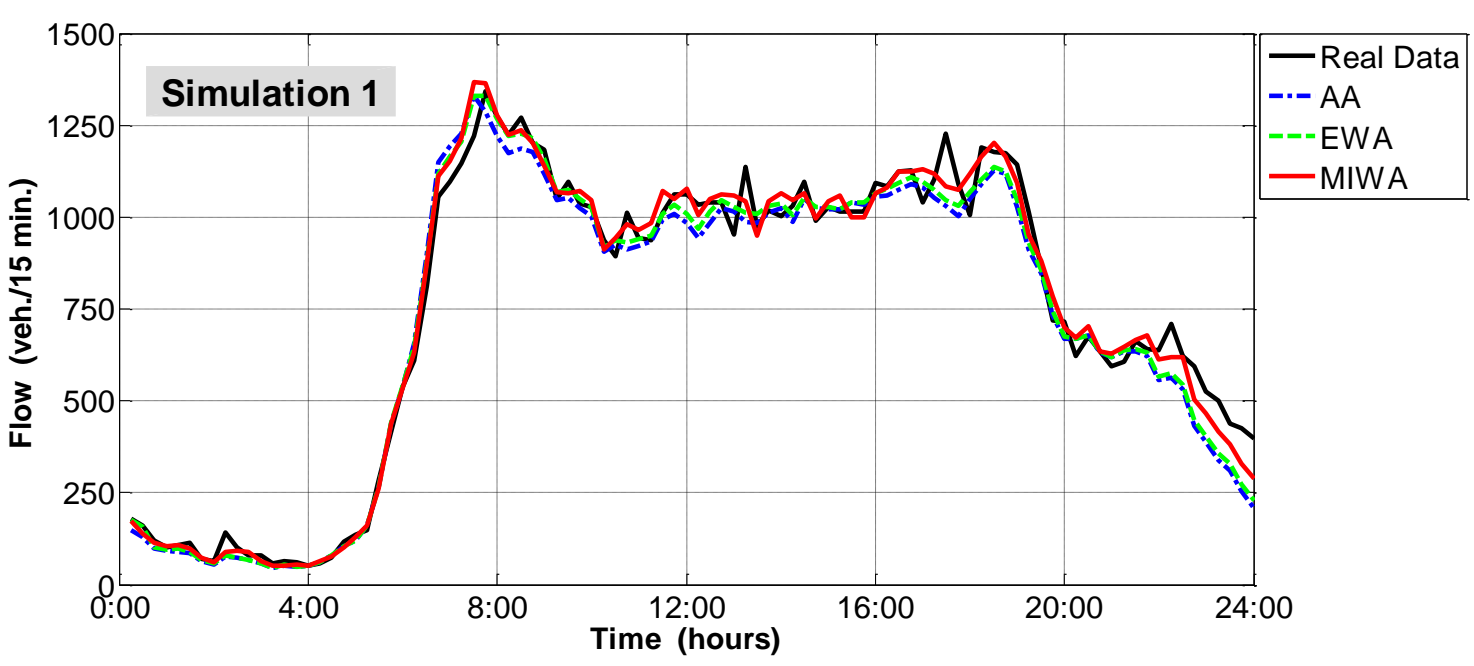

Figure 6: Traffic flow prediction for different data fusion algorithms in simulation 1 
In first simulation, we predicted traffic flow with help of normal data and evaluated performance of proposed fusion algorithms. Among fusion algorithms, because of MI algorithm utilization and effect of error amount in all prediction models on weighted factor determination of a data set, MIWA algorithm have been better in comparison with its rivals, indicating its strong ability in prediction of normal traffic flow.

\subsection{Simulation 2: Prediction using the noisy data}

Traffic flow prediction using normal data and some abnormal disturbance data (rain/snowfall or accident) along with comparison of performance between MIWA fusion algorithm and other fusion algorithms are of interest in this simulation. Hence, data time interval is presumed to be one month (April) and our aim is to predict traffic flow of Thursday, April $26^{\text {th }}$ using past 20 work days' data. One could figure out analyzing all data, probably due to sensor failure, there is no saved data in Thursday, April $19^{\text {th }}$ and all traffic flow data is zero, according to Figure 7. Likewise, Tuesday, April $3^{\text {th }}$ has also experienced such situation probably as a result of accident (http1). We aim at observing these days and noisy data including traffic conditions' change affecting prediction procedure of traffic flow. Simulation 2 procedure is like the simulation 1 and Figure 8 and Table 2 depict results of new simulation.

Table 2: Traffic flow prediction error for different data fusion algorithms in simulation 2

\begin{tabular}{c|c|c|c}
\hline \multirow{2}{*}{ Simulation 2 } & \multicolumn{3}{|c}{ Data Fusion Algorithms } \\
\cline { 2 - 4 } & AA & EWA & MIWA \\
\hline MAE & & 36.5643 & 35.0179 \\
\hline MAPE (\%) & & 7.0644 & 5.6873 \\
\hline VAPE (\%) & & 0.4554 & 0.2272 \\
\hline
\end{tabular}

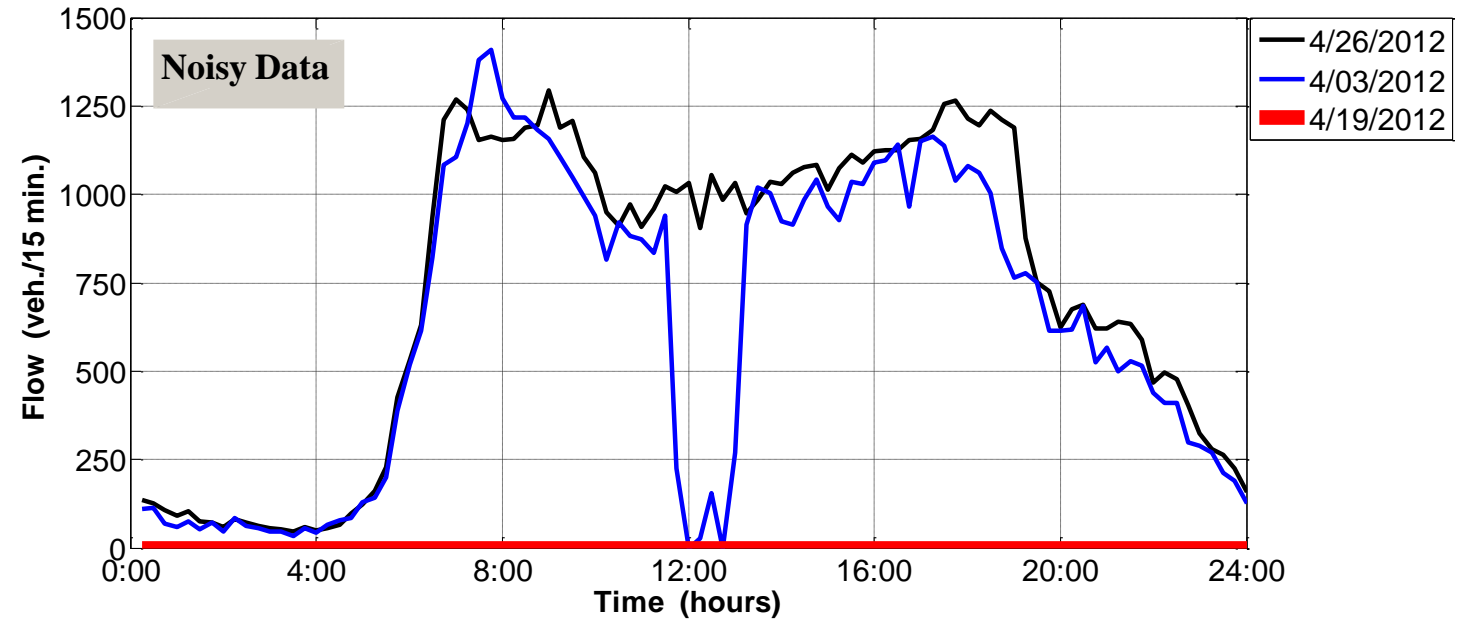

Figure 7: Traffic flow of noisy data in April

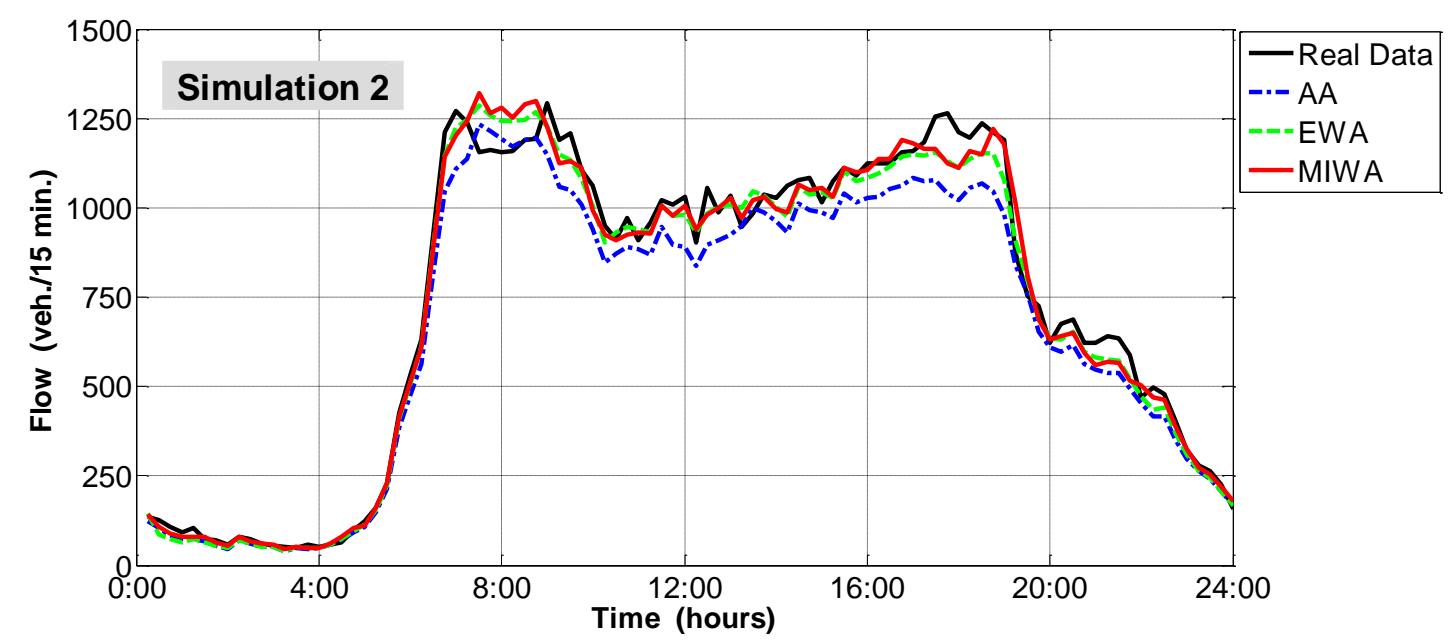

Figure 8: Traffic flow prediction for different data fusion algorithms in simulation 2 
General procedure of second simulation consists of traffic flow prediction with normal and abnormal data. Abnormal data used in this simulation are consequences of either traffic conditions (accident on the road) or sensor failure. As expected and extracted from Table 2, MIWA fusion algorithm had the best tracking performance with least prediction error, indicating its strong ability in traffic flow prediction and high resistance in presence of abnormal noisy data. As one might conclude, new proposed algorithm is less dependent to data type, recognizes defected data well and reduces their effect on prediction. MIWA algorithm is the best solution in this simulation, too.

\section{CONCLUSION}

These days, traffic flow prediction is considered one of the most important topics in intelligent transporting systems. In-depth prediction depends on the given and its corrected information. The prediction models need deeply the previous data and analyzing them which is because of the high capacity of data and slow speed of it impossible. Some prediction models are able to promisingly predict instant changes in traffic systems. These models are suitable to predict sudden traffic phenomena such as accident on the road. Some other prediction models are considered favorable to predict slowly incremental phenomena like heavy rain/snowfall on the road. Therefore, fusion models have to be capable of more accurate prediction and considering prementioned situations simultaneously. Generally, all fusion algorithms discussed in this paper are members of weighted averaging (WA) fusion models family whose weighted factors are calculated independently. Resulted weighted factors indicate value of each fusion algorithm in combination process. Considering studies in this field, using these fusion algorithms in traffic field to predict flow is presumed an innovation.

In this paper, a new fusion algorithm called MIWA was developed using mutual information algorithm in order to temporarily predict traffic flow of a-step-ahead (15 minutes later). At first, the MIWA fusion algorithm calculates the existing nonlinear dependence among the previous traffic data and infected and not corrected one is found. Then out of the large amount of given data, for decreasing the calculation volume, MIWA fusion algorithm select the best and important data. Therefore, just by selecting the given data, the prediction with high accuracy and short time is done. According to calculation structure of MIWA, it has high accuracy in comparison with various fusion algorithms (AA and EWA). Whole traffic flow prediction process was performed and simulated using real-time data related to a highway in Minnesota, USA. Real-time used data in literature include normal and disturbance (rain/snowfall or accident) data whose performance was considered in two executed simulations of traffic flow prediction. As one can see from the simulations, MIWA fusion algorithm is too sensitive to disruptive data, recognizes them and independently is able to present more accurate prediction than others.

\section{Acknowledgment}

This paper is based on work supported by the East Tehran branch of Islamic Azad University under grant MP-6508.

\section{References}

Alexandre, L., Campihlo, A., Kamel, M. (2001). On combining classifiers using sum and product rules. Pattern Recognition Letters, 22, 1283-1289.

Battiti, R. (1994). Using mutual information for selecting features in supervised neural net learning. IEEE Transactions on Neural Networks, 5, 537-550.

Bin, Y., Zhongzhen, Y., Baozhen, Y. (2006). Bus Arrival Time Prediction Using Support Vector Machines. Journal of Intelligent Transportation Systems, 10(4), 151-158.

Castro-Neto, M., Jeong, Y. S., Jeong, M. K., Han, L. D. (2009). Online-SVR for short-term traffic flow prediction under typical and atypical traffic conditions. Expert Systems with Applications, 36, 6164-6173.

Chang, H., Lee, Y., Yoon, B., Baek, S. (2012). Dynamic near-term traffic flow prediction: system-oriented approach based on past experiences. IET Intelligent Transportation Systems, 6(3), 292-305. 
Chong, C. Y. (1979). Hierarchical estimation. In Proceedings of the MIT/ONR C3 Workshop, Monterey, CA.

Durrant-Whyte, H., Stevens, M., Nettleton, E. (2001). Data fusion in decentralized sensing networks. In $4^{\text {th }}$ International Conference on Information Fusion, pp. 302-307.

Hosseini, S. H., Moshiri, B., Rahimi-Kian, A., Araabi, B. N. (2012). Short-term traffic flow forecasting by mutual information and artificial neural networks. IEEE International Conference on Industrial Technology, Athens, Greece, pp.1136-1141.

Hosseini, S. H., Moshiri, B., Rahimi-kian A., Araabi, B. N. (2014). Traffic flow prediction using MI algorithm and considering noisy and data loss conditions: An application to Minnesota traffic flow prediction. Journal PROMET-Traffic \& Transportation, 26(5), 393-403.

http1: www.d.umn.edu/tdrl/traffic/, Retrieved June 30, 2013.

http2:www.d.umn.edu/ tkwon/TDRLSoftware/T ravelTimeMetroTC.msi, Retrieved October 1, 2015.

Jiang, X., Adeli, H. (2005). Dynamic wavelet neural network model for traffic flow forecasting. Journal of Transportation Engineering, 131(10), 771-779.

Li, X. R., Zhu, Y. M., Wang J., Han, C. Z. (2003). Optimal linear estimation fusion Part I: unified fusion rules. IEEE Transactions Information Theory, 49(9), 2192-2208.

Nagare, A., Bhatia, Sh. (2012). Traffic Flow Control using Neural Network. International Journal of Applied Information Systems, 1(2), 50-52.

Ramezani, A., Moshiri, B., Rahimi-Kian, A., Aarabi, B., Abdulhai, B. (2012). Distributed maximum likelihood estimation for flow and speed density prediction in distributed traffic detectors with Gaussian mixture model assumption. IET Intelligent Transportation Systems, 6(2), 215-222.

Spyer, L. (1979). Computation and transmission requirements for a decentralized linearquadratic-gaussian control problem. IEEE Transactions Automatic Control, 24, 266-269.
Stathopoulos, A., Dimitriou, L., Tsekeris, T. (2008). Fuzzy modeling approach for combined forecasting of urban traffic flow. Computer-Aided Civil and Infrastructure Engineering, 23, 521-535.

Sun, H., Liu, H. X., Xiao, H., Ran, B. (2003). Short-term traffic forecasting using the local linear regression model. Presented at the 82nd Transportation Research Board Annual Meeting, Washington DC, pp. 1-18.

Tan, M. C., Wong, S. C., Xu, J. M., Guan, Z. R., Zhang, P. (2009). An Aggregation Approach to Short-Term Traffic Flow Prediction. IEEE Transaction on Intelligent Transportation Systems, 10(1), 60-69.

Torra, V., Narukawa, Y. (2007). Modeling Decisions Information Fusion and Aggregation Operators, Springer.

Williams, B. M. (2001). Multivariate vehicular traffic flow prediction: an evaluation of ARIMAX modeling. Transportation Research Board, 1776, 194-200.

Williams, B. M., Hoel, L. A. (2003). Modeling and Forecasting Vehicular Traffic Flow as a Seasonal ARIMA Process: Theoretical Basis and Empirical Results. Journal of Transportation Engineering, 129(6), 664-672.

Williams, J.W., Li, Y. (2009). Estimation of mutual information: A survey. In Edition Springer, Rough Sets and Knowledge Technology, pp. 389-396.

Xu, Z., Da, Q. L. (2003). An Overview of Operators for Aggregating Information. International Journal of Intelligent Systems, $18,953-969$.

Zeng, D., Xu, J., Gu, J., Liu L., Xu, G. (2008). Short term traffic flow prediction using hybrid ARIMA and ANN models. Workshop on Power Electronic and Intelligent Transportation Systems, Guangzhou, China, pp. 621-625.

Zhang, Y. (2012). How to Provide Accurate and Robust Traffic Forecasts Practically, in AbdelRahim, A. (Ed.): Intelligent Transportation Systems (INTECH, eBook), pp. 189-206. 